\title{
OPINIONES
}

\section{Necesidad de que las Fuerzas Armadas realicen un control de las plantas invasoras en el Archipiélago de Juan Fernández, Chile}

\author{
Necessity of Chilean Army to do plant invader control in Juan Fernandez Archipelago, Chile \\ Cecilia Smith-Ramírez a*, Gisella Arellano-Cataldo ${ }^{\text {a }}$

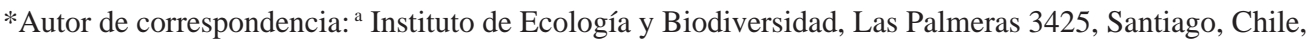 \\ tel.: 56-63-247975, csmith@willnet.cl
}

\section{SUMMARY}

\begin{abstract}
We propose that one effective way to control plant invasion in JF archipelago is to involve the Chilean Army in the process of mitigation and eradication. We call for their coordinated support in the effort to conserve the biological patrimony of the archipelago, consistent with the Line of Social and Enterprise Responsibility with the common environment. We present the background information needed to achieve success in this task and specify the work that needs to be done by the Army in the short-term to stop plant invasion, particularly around areas with critically endangered species, and in the interior and edge of endemic remnant forests.
\end{abstract}

Key words: biological conservation, plant invaders, social and enterprise responsibility.

\section{RESUMEN}

Se plantea la necesidad de que las Fuerzas Armadas de Chile realicen el control de plantas invasoras en el archipiélago de Juan Fernández. Se hace un llamado de cooperación para la conservación del patrimonio medioambiental a la línea de las Fuerzas Armadas que trata sobre responsabilidad social y empresarial, y que cuenta con experiencia en el trabajo ambiental. Se plantean antecedentes de por qué deben ser las Fuerzas Armadas quienes estén a cargo de desarrollar esta labor, sus actividades asociadas y el esfuerzo humano requerido durante los primeros años de trabajo.

Palabras clave: conservación biológica, plantas invasoras, responsabilidad social y empresarial.

\section{INTRODUCCIÓN}

La restauración ecológica en islas requiere de una primera etapa que consiste en el control o erradicación de las especies invasoras (Howald et al. 2007). A nivel mundial se han realizado 332 erradicaciones exitosas de roedores en islas (Howald et al. 2007), 48 de gatos asilvestrados (Nogales et al. 2004) y 120 de cabras (Campbell y Donlan 2005), las cuales generalmente han producido aumentos considerables del número de individuos nativos a proteger. Las islas del archipiélago Juan Fernández también requieren controlar o erradicar especies invasoras para ser restauradas. Por ejemplo, cuando el conejo europeo (Oryctolagus cuniculus Linnaeus) fue erradicado de la isla Santa Clara por la Corporación Nacional Forestal (CONAF), se produjo una rápida recuperación de la flora nativa y de fardelas (aves de la familia Procellariidae) amenazadas de extinción (Díaz 2013).

Juan Fernández se encuentra $670 \mathrm{~km}$ al oeste del continente, frente a la región de Valparaíso, Chile. Lo conforman tres islas: Santa Clara, Marinero Alejandro Selkirk y Robinson Crusoe, las cuales suman en total $147,5 \mathrm{~km}^{2}$.
Este pequeño archipiélago ha sido considerado por $\mathrm{Du}$ rell Conservation Trust, World Wildlife Fund y Bird Life International prioridad mundial de conservación, debido al alto endemismo por superficie y alta amenaza. En Robinson Crusoe y Alejandro Selkirk son plagas tres especies de roedores, cabras (Capra hircus Linnaeus) y gatos silvestres (Felis silvestris Schreber). En Robinson Crusoe además, se encuentra el coatí (Nasua nasua Linneaeus) y el conejo europeo. Más aún, en Robinson Crusoe y Alejandro Selkirk se encuentran las fitoplagas: maqui (Aristotelia chilensis (Molina) Stuntz), mora (Rubus ulmifolius Schott) y murta (Ugni molinae Turcz). Estas tres especies invasoras representan 46,6 \% de la cobertura vegetal leñosa de Robinson Crusoe y 2,2 \% de Alejandro Selkirk (Díaz 2012), manteniendo un aumento poblacional en ambas islas (Dirtbock et al. 2003, Arellano 2012, Díaz 2012).

Dada la gran envergadura del trabajo de erradicar animales invasores (costo U\$ 17-35 millones ${ }^{1}$, Saunders et al.

\footnotetext{
${ }^{1}$ Hugo Arnal. Director de International Sustainable Conservation, comunicación personal.
} 
2011) y la necesidad de controlar plantas invasoras y de proteger la biodiversidad de Juan Fernández, se plantea que una efectiva erradicación de plagas requerirá de infraestructura y dotación humana que, a nivel país, sólo se encuentra en las Fuerzas Armadas (FFAA). Se plantea que el conocimiento acumulado sobre erradicaciones por la comunidad científica internacional, con resultados exitosos en islas del mundo (Saunders et al. 2011), aunado a la infraestructura y preparación militar posibilitaría en pocos años erradicar las plagas animales y disminuir la tasa de avance de plantas invasoras.

En este trabajo se presentan antecedentes de por qué es necesaria y urgente la intervención de las FFAA de Chile para el control de fitoplagas en Juan Fernández y se propone una priorización de los sitios que requieren mayor intervención. Se enfatiza en este trabajo la necesidad de controlar fitoplagas debido a su dificultad logística y al mayor lapso requerido para obtener logros, por lo que se requiere un compromiso-país.

\section{ANTECEDENTES}

Durante doce años CONAF, "Aves Chile”, la Fundación Oikonos y miembros de la comunidad de Robinson Crusoe han estado realizando remoción de maqui y mora en el bosque de Plazoleta El Yunque. Desde el año 2006, la comunidad controla aproximadamente media hectárea al año. El control anual que hace la comunidad es cerca de $0,06 \%$, para un total de 807,7 ha, que se encuentran invadidas. La tasa potencial/anual de avance de la invasión sería de 1,1 \% para 1.015 ha de bosque remanente ${ }^{2}$. A su vez, Dirnböck et al. (2003) determinaron que de no detener el avance de las plantas invasoras, el $50 \%$ del bosque de Robinson Crusoe tendrá una alta probabilidad de ser reemplazado en un período de 80 años.

Es necesario ampliar el control mecánico (remoción) y químico de plantas invasoras en Juan Fernández, a la vez de explorar en paralelo otras alternativas de control (como el biológico). Para que el control mecánico sea eficiente en relación al avance de las plantas invasoras, este debiera ser realizado por un grupo de trabajo externo a la isla, debido a que no hay suficiente dotación humana en ésta. Este grupo de trabajo debiera ser subvencionado por el Estado y ser entrenado por los guarda-parques y el grupo local de control de plantas invasoras. Se plantea que este grupo de trabajo externo sea aportado por las FFAA actuando mediante su línea de Responsabilidad Social y Empresarial. Entrevistas hechas a tomadores de decisiones y a la comunidad de San Juan Bautista de Juan Fernández, muestran un 100 \% de apoyo a la iniciativa aquí planteada (Gutiérrez 2013).

La línea de Responsabilidad Social y Empresarial de las FFAA de Chile es considerada única entre los ejérci-

\footnotetext{
2 Smith-Ramírez C, G Arellano, JP Mora, E Hagen, R Vargas. 2012. El rol de Turdus falcklandii como dispersor de plantas invasora en el archipiélago de Juan Fernández. Revista Chilena de Historia Natural (en prensa).
}

tos del mundo (Pardow 2007). El general Oscar Izurieta señala que dicha línea de trabajo es un apoyo al desarrollo económico, social y ambiental del país. Además, el general reconoce que la profesión militar posee un carácter vocacional y valórico de los recursos naturales del país, el cual se propone sea la base del accionar en conservación de los recursos naturales amenazados por las especies invasoras. Existe la necesidad tanto de un amplio operativo militar en lugares remotos con abrupta topografía como de infraestructura de gran envergadura, de la cual carecen el Ministerio del Medio Ambiente y la CONAF (como aviones, helicópteros, buques, botes zodiacs, personal especializado en escalada, etc.). Estos recursos debieran ser provistos por el Ministerio de Defensa.

El compromiso por el bienestar del país por parte de las FFAA traspasa la estrecha visión de proyectos ambientales de financiamiento nacional e internacional, los cuales no van más allá de uno a cinco años. Es probable que en el caso de las plantas invasoras en Juan Fernández el plan de acción que se plantee implique controlar gran parte de la distribución de éstas en alrededor de una centuria. No es recomendable remover una amplia superficie de fitoplagas en la accidentada topografía del archipiélago, debido a que la cobertura del bosque se recupera lentamente, siendo la erosión la amenaza que subyace a la erradicación masiva de estas. Por ahora, se propone no remover superficies $>100 \mathrm{~m}^{2}$ en área planas, ni > $50 \mathrm{~m}^{2}$ en sitios con pendiente, y sólo hacerlo en lugares cuya geomorfología lo permita. El bosque nativo remanente de Robinson Crusoe (1.015 ha) se encuentra fragmentado por la invasión, presentando el fragmento de mayor tamaño120 ha (cuadro 1). $\mathrm{Al}$ interior de los fragmentos existen numerosos claros que han sido ocupados por las especies invasoras. A partir de los resultados positivos de recuperación del bosque en Plazoleta (Vargas et al. 2013), se recomienda que un trabajo similar sea hecho en otras áreas de bosque en Robinson Crusoe.

La Fundación Oikonos calcula que un equipo de cinco personas controla una hectárea de maqui y mora en 3,7-8,5 meses. La variación en la eficiencia de trabajo depende de la accesibilidad, clima y supervisión. Según señala Paola González de Rodt, líder del equipo de control de malezas, la eficiencia del equipo de trabajo de cinco personas puede aumentar a una hectárea controlada en dos meses, con adecuada supervisión.

\section{DÓNDE, EN CUÁNTO TIEMPO Y CUÁNTAS PERSONAS}

No hay consenso de dónde empezar a controlar, debido a la urgencia de proteger muchos lugares a la vez. Sin embargo, existe consenso en que deben ser controladas las plantas invasoras alrededor de las poblaciones de plantas en Estado Crítico de Extinción, que presenten menos de 500 individuos y estén amenazadas por la expansión de estas.

Primero, debe ponerse especial atención al control de invasoras en la cima del cerro el Yunque, sitio inaccesible 
Cuadro 1. Superficies de los fragmentos de bosque remanentes en la matriz de plantas invasoras, pastizales y suelo desnudo.

\begin{tabular}{crc}
$\begin{array}{c}\text { and bare soil. } \\
\text { Rango de } \\
\text { tamaños (ha) }\end{array}$ & $\mathrm{N}^{\text {o } \text { fragmentos }}$ & $\begin{array}{c}\text { Superficie } \\
\text { acumulada (ha) }\end{array}$ \\
\hline$<0,1$ & $<1.000$ & 155,7 \\
$0,1-1,0$ & 151 & 65,3 \\
$1,1-5,0$ & 43 & 90,9 \\
$5,1-15,0$ & 11 & 115,8 \\
$15,1-40,0$ & 12 & 297,3 \\
$40,1-120,0$ & 3 & 290,0 \\
\hline Total & $<1.220$ & $1.015,0$ \\
\hline
\end{tabular}

para personas sin conocimiento en técnicas de escalada ${ }^{3}$. De no hacerse un control de especies invasoras en este lugar se espera la novena extinción en el archipiélago, esto es, la especie de género endémico Yunquea tenzii Skottsb.

Segundo, debido a que la capacidad de nucleación del bosque se pierde en la medida que la invasión de maqui y mora lo desintegran (cuadro 1), se propone que se priorice controlar al interior de los fragmentos de bosques. La comunidad fernandeziana propone que se realicen ensayos de control de especies invasoras en distintos lugares de la isla, de tal forma de poder evaluar la recuperación del bosque por sector.

A continuación se entregan estimaciones de número de personal requerido para el control de plantas invasoras, considerando que una hectárea de malezas puede ser removida cada dos meses por un equipo de cinco personas.

Año 1. Probablemente 2/3 de las plantas en peligro crítico de Robinson Crusoe (de un total de 21) se encuentren en acantilados, protegidas del ramoneo de animales y de las plantas invasoras, pero aproximadamente $1 / 3$ se encontrarían bajo la cobertura de éstas. Alrededor de estas poblaciones debiera generarse un área de amortiguación libre de plantas invasoras. Es posible que un equipo de cinco personas por día pueda generar dicha área de amortiguación, por cada población de planta amenazada. Es de considerar que la mayor parte del tiempo se ocupará en llegar al sitio de trabajo. Se estima que un equipo de cinco personas trabajando durante dos meses podrá lograr este objetivo. Posterior a la remoción, en algunos sectores será necesario implementar medidas de control de erosión. Estas mismas áreas de control pueden servir de parcelas de ensayo para determinar la tasa de recuperación del bosque como ha sido propuesto por la comunidad.

También es necesario rescatar gran parte de los 43 fragmentos remanentes entre 1,1 a 5,0 ha que quedan inmersos en la matriz de maqui y mora (cuadro 1 ), que tengan

\footnotetext{
${ }^{3}$ Iván Leiva. Administrador del Parque Nacional Juan Fernández, comunicación personal.
}

menos dificultad de acceso y por ende de mantenimiento, y que presenten características que aumenten su valor de conservación, por ejemplo, presencia de plantas amenazadas. Este rescate debiera hacerse controlando al interior de los fragmentos y generando un área de amortiguación alrededor de cada uno. El ancho del área de amortiguación dependerá de la pendiente. En general, la accesibilidad al bosque es difícil, por lo cual es posible calcular tres días promedio de trabajo efectivo por cada fragmento; en total cerca de cinco meses serían necesarios para que un equipo de cinco personas haga esta labor. Debido a que los meses más favorables para realizar control de plantas invasoras ocurren de diciembre a marzo, se recomienda contar con dos equipos de cinco personas trabajando a la vez. Por lo cual, durante el primer año se solicita a las FFAA de Chile su apoyo en el traslado y mantención de 15 personas las cuales trabajarían durante tres meses del período estival.

Años 2 al 6. Se propone que las prioridades de control los años 2 al 6 sea en los 26 fragmentos de bosque remanente mayores a 5 ha. Actualmente quedan cinco fragmentos de bosque nativo en Vaquería, cuatro en La Piña-Puerto Francés, cuatro en Villagra, tres en el Mirador de Selkirk, tres en Plazoleta, dos en Puerto Inglés, uno en Damajuana y uno en La Pascua, todos inmersos en la matriz de maqui, mora y murta, pastizales y suelo desnudo. Se propone en el segundo año rescatar estos fragmentos, empezando desde los más pequeños a los más grandes. Se desconoce la superficie total posible de controlar en estos fragmentos de bosque endémico, sin embargo, a partir del esfuerzo hecho en Plazoleta, bosque de tamaño relativamente similar a la mayoría de los ya señalados, se estima que este objetivo podría ser cumplido en cinco años de trabajo (cuatro meses por año, por seis equipos de cinco personas). A partir de los resultados de avance del año 1 se puede re-calcular el número de personal necesario, pero se recomienda no trabajar con más de 30 personas a la vez, debido a la compleja logística de la isla.

Cada año las prioridades de dónde controlar deben ser re-evaluadas y en todos los casos incluir cada dos años la mantención de los sitios controlados anteriormente.

En los años siguientes. Habiendo sido realizado el control de plantas invasoras en los sitios prioritarios, en los años siguientes este control debiera centrarse en la mantención de las áreas ya controladas, avanzando desde las áreas menos invadidas hacia la matriz invasora, estrategia de manejo recomendada en ambientes altamente invadidos (Mack y Moody 1992). Para realizar este tipo de control se requiere de un manejo adaptativo, esto es, cada vez que el equipo de trabajo se enfrente a un nuevo escenario se debe evaluar el éxito de la medida a realizar y buscar alternativas en caso de no ser aceptable el plan predefinido.

El control de plantas invasoras en Juan Fernández, debe ser un proceso lento, para evitar la erosión, pero no menor que la tasa de avance anual de las mismas. Así, si el 
avance de las plantas invasoras es cerca de 11 ha por año $0^{4}$, cada año debiera controlarse un poco más de esta cantidad, sino se está en un círculo vicioso que no llevará al éxito de las medidas tomadas.

Es de notar que no se ha hecho mención sobre la necesidad (según la CONAF) de controlar y, de ser posible, erradicar los tres arbustos invasores en Alejandro Selkirk. En esta isla existirían 139 ha de plantas invasoras (CONAF 2010), representadas especialmente por maqui. Los lugares de difícil acceso en Alejandro Selkirk como quebrada Las Casas, donde se encuentra gran parte de la población de maqui, hacen imperioso que personal experto en escalada enfrente esta tarea.

\section{CONCLUSIONES}

Sólo un proyecto a nivel país que involucre décadas de trabajo podrá salvar la biodiversidad del archipiélago de Juan Fernández de lo que parece ser su inminente colapso a corto plazo producto de la acción de las especies invasoras, especialmente, de plantas. Probablemente, sólo la intervención de un grupo altamente organizado como las FFAA de Chile, con la ayuda de CONAF, Ministerio del Medio Ambiente, lugareños y científicos podrá detener la sentencia que pesa sobre una de las mayores joyas de la biodiversidad mundial.

\section{AGRADECIMIENTOS}

Agradecemos a CONAF, la comunidad de Juan Fernández y a dos revisores anónimos.

\section{REFERENCIAS}

Arellano G. 2012. Evaluación de la dinámica de invasión de Aristotelia chilensis (Elaeocarpaceae) y Rubus ulmifolius (Rosaceae) en claros de dosel en un bosque de la isla Robinson Crusoe, Archipiélago de Juan Fernández, Chile. Tesis de magíster. Santiago, Chile. Facultad de Ciencias Forestales y Conservación de la Naturaleza, Universidad de Chile. 63 p.
Campbell K, J Donlan. 2005. Feral goat eradications on islands. Conservation Biology 19(5): 1362-1374.

CONAF (Corporacion Nacional Forestal, CL). 2010. Plan de manejo del Parque Nacional Juan Fernández. Valparaíso, Chile. CONAF. 128 p.

Díaz I. 2013. Análisis de la evolución espacio-temporal de la invasión de Rubus ulmifolius, Aristotelia chilensis y Ugni molinae en la Isla Robinson Crusoe. Tesis Ingeniero en Recursos Naturales. Santiago, Chile. Facultad de Ciencias Agronómicas, Universidad de Chile. 53 p.

Dirnböck T, J Greimler, P López, TF Stuessy. 2003. Predicting future threats to the native vegetation of Robinson Crusoe Island, Juan Fernandez Archipelago, Chile. Conservation Biology 17(3): 1650-1659.

Gutiérrez H. 2013. Percepción y propuesta de la comunidad del archipiélago de Juan Fernández sobre las amenazas a su biodiversidad y la erradicación y/o control de especies exóticas invasoras. Santiago, Chile. Tesis de Agronomía. Facultad de Agronomía, Universidad de Chile. 45 p.

Howald G, CJ Donlan, JP Galvan, JC Russell, J Parkes, A Samaniego, Y Wang, D Veitch, P Genovesi, M Pascal, A Saunders, B Thershy. 2007. Invasive rodent eradication on islands. Conservation Biology 21(2): 1258-1268.

Mack RN, ME Moody. 1992. Modelling to predict the fate of invading plants. In Stone CP, CW Smith, JT Tunison eds. Alien Plant Invasions in Native Ecosystems of Hawai'i. Univ. Hawaii Cooperative National Park Resources Studies Unit. Honolulu, USA. Univ. Hawaii Press. p. 739-742.

Nogales M, A Martín, BR Tershy, CJ Donlan, D Veitch, N Puerta, J Alonso. 2004. A review of feral cat eradication on islands. Conservation Biology 18(2): 310-319.

Pardow R. 2007. Reporte de responsabilidad social del Ejército de Chile, un compromiso con la sociedad. Santiago, Chile. Ejército de Chile. 6 p.

Saunders A, A Glen, K Campbell, R Atkinson, J Sawyer, E Hagen, H Torres.2011. Feasibility of managing invasive species in the Juan Fernández Archipelago, Chile. Lincoln, New Zealand. Invasive Species International. 156 p.

Vargas R, C Smith-Ramírez, C González, M Fernández. 2013. El archipiélago de Juan Fernández, endemismo para conservar. In Moreira-Muñoz A, A Borsdorf eds. Reservas de la Biosfera de Chile: naturaleza y ser humano. Instituto de Geografía UC. Academia de Ciencias Austria, Corporación Taller La Era. 162 p. 